\title{
QUOTIENTS OF COMPLEX MANIFOLDS AND MODULI SPACES OF KLEIN SURFACES*
}

\author{
MIKA SEPP ̈̈L $\ddot{A} * *$ \\ Dedicated to the memory of Aldo Andreotti
}

\section{Introduction}

N. L. Alling and N. Greenleaf showed in 1969 ([1]) that the analytic counterpart of a real algebraic function field in one variable is a compact Klein surface, i.e., a compact surface, which may be non-orientable and may have boundary, endowed with a dianalytic structure. This observation leads us to study moduli spaces of Klein surfaces.

Since the Riemann sphere has a unique analytic structure, all Klein surfaces of genus 0 have a unique dianalytic structure. Hence the case $g=0$ is trivial. The special case of Klein surfaces of genus 1 can be handled with explicit methods. N. L. Alling has obtained good results that fully characterize the moduli spaces of Klein surfaces of genus 1 (cf. [2] and the forthcoming book on elliptic curves by Alling). Here we study only the general case $g>1$.

In his paper "On the moduli of closed Riemann surfaces with symmetries" C. J. Earle studied Teichmüller spaces of symmetric Riemann surfaces. In a special case those Teichmüller spaces are exactly the same as Teichmüller spaces of Klein surfaces as defined in [6]. In order to study moduli spaces of symmetric Riemann surfaces Earle defined certain $\bmod n$ relative Teichmüller spaces. In the case of Klein surfaces, however, one can proceed directly and define a real analytic structure on the moduli space $X(Z)$ of Klein surfaces of a given topological type. In this paper that is done in Section 5.

For the considerations in Section 5 we need auxiliary results concerning quotients of complex manifolds with a real structure. Following A. Andreotti and P. Holm we say that an antiholomorphic involution of a complex manifold $A$ is a real structure on $A$ ([3]). In Section 1 we show that if a group $G$ of holomorphic

* This is an expanded version of the lectures given at Columbia University in New York and at the University of Rochester in September 1979.

** Work supported by the Finnish Cultural Foundation. 
automorphisms of a complex manifold $A$ acts properly discontinuously on $A$, the quotient $\bmod G$ of a real analytic subset $B$ of $A$ is a real analytic subset of $A / G$ provided that the group $G$ keeps $B$ fixed (as a set). Using that result we can easily study the quotient of the real part of a complex manifold with a real structure (Theorem 2).

In Section 6 we consider the subspace of the moduli space $X\left(Z_{c}\right)$ of Riemann surfaces of genus $g(>1)$ which consists of points corresponding to Riemann surfaces that have antiholomorphic involutions. This is the same as the subspace of the moduli space of complex curves of genus $g$ whose points can be defined by real polynomials. In the main theorem of this work (Theorem 5) we show that this is a real analytic subspace which is contained in the quasiregular real part of the moduli space $X\left(Z_{c}\right)$ (it has a natural real structure) and that if $g>2$, its irreducible components correspond to Klein surfaces of a given topological type. If the genus is at least 4 , we can show that the above subspace coincides with the quasiregular real part of the moduli space $X\left(Z_{c}\right)$.

The author wishes to dedicate this paper to the memory of Aldo Andreotti whose help was essential for its completion. The mathematical community is much poorer for the absence of a man whose ideas influenced it so broadly and deeply. The author feels even greater loss, that of a friend and mentor.

I am also grateful to Clifford Earle who corrected a mistake and made many other useful remarks.

\section{Quotients of real analytic subsets of a complex manifold}

Let $A$ be a fixed connected complex manifold, and let $B \subset A$ be a real analytic subset. Consider a group $G$ of holomorphic automorphisms of $A$. Assume that it acts properly discontinuously on $A$ and that each of its elements maps $B$ onto itself.

The quotient $A / G=X$ is an irreducible normal complex space. Let pr: $A \rightarrow X$ be the projection.

Theorem 1. The projection of the set $B, \operatorname{pr}(B)=B / G$, is a real analytic subset of $X$.

Proof. Let $a \in A$, and let $G_{a}=\{g \in G \mid g(a)=a\}$. By the discontinuity of $G, G_{a}$ is always a finite group. Let $G_{a}=\left\{\mathrm{Id}, g_{1}, g_{2}, \ldots, g_{n}\right\}$, where Id denotes the identity mapping of $A$.

We can find an open neighborhood $U$ of the point $a$ such that

(1) for every $g \in G_{a} \quad g(U)=U$, and for every $g \in G \backslash G_{a} \quad g(U) \cap U=\emptyset$.

(To find $U$ satisfying (1), take $V$ small enough and put $U=\cup_{g \in G_{a}} g(V)$.) 
Restricting $U$, if necessary, we can find real analytic functions $f_{i}: U \rightarrow \boldsymbol{R}$, $i=1,2, \ldots, k$, for which $B \cap U=\left\{p \in U \mid f_{i}(p)=0, i=1,2, \ldots, k\right\}$. Then $\tilde{h}, \tilde{h}(p)=$ $\sum_{i=1}^{k} f_{i}(p)^{2}$, is a real analytic function whose zero-locus in $U$ is exactly $B \cap U$. Define yet another function $\tilde{h}^{G}$, with the same property, setting

$$
\tilde{h}^{G}(p)=\tilde{h}(p) \tilde{h}\left(g_{1}(p)\right) \tilde{h}\left(g_{2}(p)\right) \ldots \tilde{h}\left(g_{n}(p)\right) .
$$

Then $\tilde{h}^{G}: U \rightarrow \boldsymbol{R}$ is real aralytic, and $\tilde{h}^{G} \circ g=\tilde{h}^{G}$ fcr all $g \in G_{a}$. It follows that $\tilde{h}^{G}$ induces a real aralytic furction $h: \operatorname{pr}(U) \rightarrow R$, for which

$$
\operatorname{pr}(B \cap U)=\{p \in \operatorname{pr}(U) \mid h(p)=0\} .
$$

By condition (1), $\operatorname{pr}(B \cap U)=\operatorname{pr}(B) \cap \operatorname{pr}(U)$. Hence $\operatorname{pr}(B) \cap \operatorname{pr}(U)$ is a real analytic subset of $\operatorname{pr}(U)$. Consequently, $\operatorname{pr}(B)$ is a real analytic subset of $X$. The theorem is proved.

Using condition (1) in the same manner as in the previous proof we get immediately:

Proposition 1. If the germ $(B, a)$ of the set $B$ at a point $a \in B$ is irreducible, then also $(\operatorname{pr}(B), \operatorname{pr}(a))$ is irreducible.

\section{Real structures on $A$}

Let $\sigma: A \rightarrow A$ be an antiholomorphic involution, i.e., $\sigma^{2}$ is the identity mapping of $A$. Assume that $\sigma$ commutes with $G$, i.e., for every $g \in G$ there is an $g^{\prime} \in G$ such that $\sigma \circ g=g^{\prime} \circ \sigma$. Then $\sigma$ induces an antiholomorphic involution $\tau: X \rightarrow X$ of the quotient space $X=A / G$ for which $\operatorname{pr} \circ \sigma=\tau \circ$ pr.

Adopting the terminology used by Andreotti and Holm ([3]) we call $\sigma$ and $\tau$ real structures on $A$ and $X$, respectively. Since $\operatorname{pr} \circ \sigma=\tau \circ \mathrm{pr}, \mathrm{pr}: A \rightarrow X$ is a real holomorphic mapping ([3], 1.2).

The fixed-point set of $\sigma, A_{\sigma}$, is called the real part of $A$. Likewise, $X_{\tau}=$ $\{p \in X \mid \tau(p)=p\}$ is the real part of $X$.

If $A_{\sigma}$ is not empty, it is a real analytic submanifold of $A$ by Proposition (1.3) in [3], and

$$
\operatorname{dim}_{R} A_{\sigma}=\operatorname{dim}_{C} A .
$$

This means that $A$ is a faithful complexification of $A_{\sigma}$ (cf. [3], 1.5).

Let $F \subset A$ be the set of fixed points of non-identity elements of $G$. The set $F$ is clearly closed.

Lemma 1. The intersection $A_{\sigma} \cap F$ is nowhere dense in $A_{\sigma}$, i.e., $A_{\sigma}$ is the closure of $A_{\sigma} \backslash F$.

Proof. Let $p \in A_{\sigma} \cap F$. Let us assume that there exists an open set $U \subset A$, $p \in U$, such that $A_{\sigma} \cap U \subset F$, and let us show that this is not possible. 
Let $G_{p}=\left\{\mathrm{Id}, g_{1}, g_{2}, \ldots, g_{n}\right\}$ be the stabilizer of the point $p$. Restricting $U$, if necessary, we find, by the discontinuity of $G$, that $A_{\sigma} \cap U$ is the fixed-point set of non-identity elements of $G_{p}$. A topological argument then shows that some non-identity element of $G_{p}$, say $g$, must fix a whole non-empty open subset of $A_{\sigma} \cap F$. But since $A$ is a faithful complexification of $A_{\sigma}$, it follows, by Corollary (7.6) in [3], that $g$ must keep an open set of $A$ point-wise fixed. Using analytic continuation we deduce then that $g$ is the identity mapping of $A$. This is a contradiction which proves the lemma.

\section{Quotients of real parts}

Consider the quotient space $X=A / G$. Let pr: $A \rightarrow X$ again denote the projection. If each element of $G$ maps the real part $A_{\sigma}$ of $A$ onto itself, we can apply Theorem 1 and deduce that $\operatorname{pr}\left(A_{\sigma}\right)$ is a real analytic subset of $X$. In general, however, the elements of $G$ do not map $A_{\sigma}$ onto itself. If such is the case, consider the set $B=\cup_{g \in G} g\left(A_{\sigma}\right)$. Each element of $G$ maps $B$ onto itself, and $\operatorname{pr}\left(A_{\sigma}\right)=\operatorname{pr}(B)$.

Let us prove that $B$ is a real analytic subset of $A$. Since the mappings $g \in G$ are holomorphic automorphisms of $A$, each set $g\left(A_{\sigma}\right)$ is a real analytic subset of $A$. Hence it suffices to show that each point $p \in A$ has an open neighborhood $U$ such that $g\left(A_{\sigma}\right) \cap U \neq \emptyset$ for at most a finite number of different sets $g\left(A_{\sigma}\right)$.

In order to find such an $U$, let $V$ be an open neighborhood of the point $p$, and assume that the closure $\bar{V}$ of $V$ is compact. If none of the sets $g\left(A_{\sigma}\right)$ intersect $V$, we have nothing to show. If that is not the case, assume that $g_{0}\left(A_{\sigma}\right) \cap V \neq \emptyset$. The mapping $\beta=g_{0} \circ \sigma \circ g_{0}^{-1}$ is an antiholomorphic involution of $A$, and $A_{\beta}=g_{0}\left(A_{\sigma}\right)$. The set $U=V \cup \beta(V)$ is an open neighborhood of the point $p$, and the closure of $U$ is compact. Since $G$ is properly discontinuous, $g(U) \cap U \neq \emptyset$ for only a finite number of mappings $g \in G$.

Assume that $g\left(A_{\sigma}\right) \cap U \neq 0$. Then $g \circ \sigma \circ g^{-1} \circ \beta \in G$ and $g \circ \sigma \circ g^{-1} \circ \beta(U) \cap$ $U \neq \emptyset$. Hence there can be only a finite number of different elements of the form $g \circ \sigma \circ g^{-1}$, where $g \in G$ is such that $g\left(A_{\sigma}\right) \cap U \neq 0$.

Assume that $g_{1} \circ \sigma \circ g_{1}^{-1}=g_{2} \circ \sigma \circ g_{2}^{-1}$. Then $\left(g_{2}^{-1} \circ g_{1}\right) \circ \sigma=\sigma \circ\left(g_{2}^{-1} \circ g_{1}\right)$. Hence the mapping $g_{2}^{-1} \circ g_{1}$ maps $A_{\sigma}$ onto itself. It follows that $g_{1}\left(A_{\sigma}\right)=g_{2}\left(A_{\sigma}\right)$, since $g_{1}=g_{2} \circ\left(g_{2}^{-1} \circ g_{1}\right)$. Consequently, only a finite number of different sets $g\left(A_{\sigma}\right)$, $g \in G$, intersect $U$. Hence $B$ is a real analytic subset of $A$.

Now we can use Theorem 1 and deduce that $\operatorname{pr}\left(A_{\sigma}\right)=\operatorname{pr}(B)$ is a real analytic subset of $X$.

The projection $\operatorname{pr}\left(A_{\sigma}\right)$ of $A_{\sigma}$ is the closure of its regular part. To prove this note first that the singular set $S$ of the normal complex space $X=A / G$ is contained in the image $\operatorname{pr}(F)$ of the fixed-point set of $G$. Since the projection 
mapping is continuous, it follows, from Lemma 1, that

$$
\operatorname{pr}(A) \subset \overline{\operatorname{pr}\left(A_{\sigma} \backslash F\right)},
$$

where the bar denotes the closure.

Since the elements of $G$ map $F$ onto itself and $A \backslash F$ onto itself, we have $\operatorname{pr}\left(A_{\sigma} \backslash F\right)=\operatorname{pr}\left(A_{\sigma}\right) \backslash \operatorname{pr}(F)$. Hence

$$
\operatorname{pr}\left(A_{\sigma}\right) \subset \overline{\operatorname{pr}\left(A_{\sigma}\right) \backslash \operatorname{pr}(F)} \subset \overline{\operatorname{pr}\left(A_{\sigma}\right) \backslash S} .
$$

On the other hand, it is clear that $\operatorname{pr}\left(A_{\sigma}\right)$ is contained in the real part $X_{\tau}$ of $X$; here $\tau$ is the involution of $X$ induced by $\sigma$. Using then Corollary (5.3) in [3], we deduce that $\operatorname{pr}\left(A_{\sigma}\right)$ is contained in the quasiregular real part $\hat{X}_{\tau}$ of $X$ Recall that the quasiregular real part $\hat{X}_{\tau}$ of $X$ consists of those points $p$ of $X_{\tau}$ for which

$$
\operatorname{dim}_{R}\left(X_{\tau}, p\right)=\operatorname{dim}_{C}(X, p),
$$

and that Corollary (5.3) in [3] simply states that $\hat{X}_{\tau}=\overline{X_{\tau} \backslash S}$.

Note that using (2), the properties of the projection and the above cited Corollary (5.3) of [3], we get

for all $p \in \operatorname{pr}\left(A_{\sigma}\right)$.

$$
\operatorname{dim}_{R}\left(\operatorname{pr}\left(A_{\sigma}\right), p\right)=\operatorname{dim}_{C}(X, p)
$$

Theorem 2. The image $\operatorname{pr}\left(A_{\sigma}\right)$ of the real part of $A$ under the projection pr: $A \rightarrow X=A / G$ is a real analytic subset of $X$. Furthermore, $\operatorname{pr}\left(A_{\sigma}\right)$ is contained in the quasiregular real part $\hat{X}_{\tau}$ of $X$, and if $A_{\sigma}$ is connected, $\operatorname{pr}\left(A_{\sigma}\right)$ is irreducible.

Proof. The only point that is not proved yet is that $A_{\sigma}$ being connected, $\operatorname{pr}\left(A_{\sigma}\right)$ is irreducible. To prove this assume that $\operatorname{pr}\left(A_{\sigma}\right)=W_{1} \cup W_{2}$, where $W_{1}$ and $W_{2}$ are real analytic subsets of $X$. Since the mapping pr: $A \rightarrow X$ is holomorphic, the preimages $\operatorname{pr}^{-1}\left(W_{1}\right)$ and $\operatorname{pr}^{-1}\left(W_{2}\right)$ are real analytic subsets of $A$. Hence also $A_{\sigma} \cap \mathrm{pr}^{-1}\left(W_{i}\right), i=1,2$, are real analytic subsets of $A$, and

$$
A_{\sigma}=\left(A_{\sigma} \cap \operatorname{pr}^{-1}\left(W_{1}\right)\right) \cup\left(A_{\sigma} \cap \operatorname{pr}^{-1}\left(W_{2}\right)\right) \text {. }
$$

Since $A_{\sigma}$ is a connected real analytic submanifold of $A, A_{\sigma}$ is irreducible Hence either $A_{\sigma} \subset \mathrm{pr}^{-1}\left(W_{1}\right)$ or $A_{\sigma} \subset \mathrm{pr}^{-1}\left(W_{2}\right)$. It follows that either $\operatorname{pr}\left(A_{\sigma}\right)=W_{1}$ or $\operatorname{pr}\left(A_{\sigma}\right)=W_{2}$. Consequently, $\operatorname{pr}\left(A_{\sigma}\right)$ is irreducible. The proof is complete.

\section{Teichmüller spaces and moduli spaces of Klein surfaces}

Theorem 2 has an interesting application in the theory of Klein surfaces. In order to understand that let us first recall some definitions and known results.

A possibly non-orientable topological surface $Z$ together with a dianalytic structure $X$ is called a Klein surface (cf. [2]). We allow $Z$ to have boundary, 
but we assume that $Z$ is connected. For a notational convenience the Klein surface $(Z, X)$ will be denoted only by $X$ where no confusion is possible.

The Klein surface $X$ is classical if it is orientable and does not have boundary, i.e., if it is a Riemann surface. Otherwise $X$ is called non-classical.

Let us now fix the topological surface $Z$; all Klein surfaces that we shall consider below will be just $Z$ with some dianalytic structure. We shall assume that Klein surfaces $(Z, X)$ are non-classical and compact. Let us also assume that the algebraic genus $g$ of Klein surfaces $(Z, X)$ is at least 2 .

We can construct a triple $\left(Z_{c}, \pi, \sigma\right)$ with the following properties:

(i) $Z_{c}$ is a compact oriented surface without boundary,

(ii) $\sigma: Z_{c} \rightarrow Z_{c}$ is an orientation reversing involution, and

(iii) $\pi: Z_{c} \rightarrow Z$ is a double covering map that satisfies $\pi \circ \sigma=\pi$.

Note that $\pi: Z_{c} \rightarrow Z$ is not a covering in the topological sense since it is a folding along $\partial Z$ (cf. [2], p. 37).

For each dianalytic structure $X$ of $Z$ we can find a unique analytic structure $X_{c}$ of $Z_{c}$ which agrees with the orientation of $Z_{c}$ and for which $\sigma: X_{c} \rightarrow X_{c}$ is all antiholomorphic involution and $\pi: X_{c} \rightarrow X$ is a dianalytic mapping (cf. [2], Proposition 1.6.2). The Riemann surface $X_{c}$ is called the complex double of $X$.

Let $K(Z)$ be the set of dianalytic structures on $Z$. Denote by $\operatorname{Hom}(Z)$ the group of homeomorphic self-mappings of $Z$, and let $\operatorname{Hom}_{0}(Z)$ be the subgroup of Hom $(Z)$ that consists of mappings homotopic to the identity mapping of $Z$. The quotient $\Gamma(Z)=\operatorname{Hom}(Z) / \operatorname{Hom}_{0}(Z)$ is called the modular group of $Z$ (or the mapping class group of $Z$ ).

The group $\operatorname{Hom}(Z)$ acts on $K(Z)$ in the following way: if $h \in \operatorname{Hom}(Z)$ and $X \in K(Z)$, then $h^{*}(X)$ is the dianalytic structure of $Z$ for which the mapping $h: X \rightarrow h^{*}(X)$ is dianalytic.

The Teichmüller space of $Z, T(Z)$, is the quotient

$$
T(Z)=K(Z) / \operatorname{Hom}_{0}(Z),
$$

and the moduli space of $Z, X(Z)$, is

By definitions,

$$
X(Z)=K(Z) / \operatorname{Hom}(Z) .
$$

$$
X(Z)=T(Z) / \Gamma(Z) .
$$

The classical definitions for Teichmüller and moduli spaces of $Z_{c}$, which is orientable and does not have boundary, are slightly different. Let $\operatorname{Hom}^{+}\left(Z_{c}\right)$ be the group of orientation preserving homeomorphic self-mappings of $Z_{c}$, while $\operatorname{Hom}_{0}^{+}\left(Z_{c}\right)=\operatorname{Hom}^{+}\left(Z_{c}\right) \cap \operatorname{Hom}_{0}\left(Z_{c}\right)$, and $\Gamma^{+}\left(Z_{c}\right)=\operatorname{Hom}^{+}\left(Z_{c}\right) / \operatorname{Hom}_{0}^{+}\left(Z_{c}\right)$. Note that, in our case, $\operatorname{Hom}_{0}^{+}\left(Z_{c}\right)=\operatorname{Hom}_{0}\left(Z_{c}\right)$. Consider the set $K^{+}\left(Z_{c}\right)$ of the analytic structures of $Z_{c}$ which agree with the orientation of $Z_{c}$. The group $\operatorname{Hom}\left(Z_{c}\right)$ still acts in $K^{+}\left(Z_{c}\right)$; if $h \in \operatorname{Hom}\left(Z_{c}\right)$ is an orientation reversing mapping, define $h^{*}(Y)$ as the analytic structure of $Z_{c}$ for which the mapping $h: Y \rightarrow h^{*}(Y)$ is antiholomorphic, $Y \in K^{+}\left(Z_{c}\right)$. Note that if $h, g \in \operatorname{Hom}\left(Z_{c}\right),(h \circ g)^{*}=h^{*} \circ g^{*}$. 
Now we can define the Teichmüller space, $T\left(Z_{c}\right)$, and the moduli space, $X\left(Z_{c}\right)$, of $Z_{c}$ in the usual manner:

$$
T\left(Z_{c}\right)=K^{+}\left(Z_{c}\right) / \operatorname{Hom}_{0}^{+}\left(Z_{c}\right), \quad X\left(Z_{c}\right)=K^{+}\left(Z_{c}\right) / \operatorname{Hom}^{+}\left(Z_{c}\right) .
$$

By definitions,

$$
X\left(Z_{c}\right)=T\left(Z_{c}\right) / \Gamma^{+}\left(Z_{c}\right) .
$$

The mapping $\pi: Z_{c} \rightarrow Z$ induces a mapping $\pi^{*}: K(Z) \rightarrow K^{+}\left(Z_{c}\right), \pi^{*}(X)=X_{c}$. It is easy to see that setting $\pi^{*}([X])=\left[X_{c}\right]$, for $[X] \in T(Z)$, we obtain a well-defined mapping $\pi^{*}: T(Z) \rightarrow T\left(Z_{c}\right)$. Let us recall the following result ([6], Theorem 5.1).

Theorem. The mapping $\pi^{*}: T(Z) \rightarrow T\left(Z_{c}\right)$ is one-to-one, and if $\sigma^{*}$ is the self-mapping of $T\left(Z_{c}\right)$ induced by the involution $\sigma$ of $Z_{c}$, then $\pi^{*}(T(Z))$ is the fixed-point set $T\left(Z_{c}\right)_{\sigma^{*}}$ of $\sigma^{*}$.

There is a natural way to define a complex structure on $T\left(Z_{c}\right)$. Since we have assumed that the genus $g$ of $Z$ is at least 2, the same applies to the genus of $Z_{c}$. Hence $T\left(Z_{c}\right)$ with that complex structure is a $3 g$-3-dimensional complex manifold.

Royden has proved that the modular group $\Gamma^{+}\left(Z_{c}\right)$ is the full group of holomorphic automorphisms of $T\left(Z_{c}\right)$. By means of that result it is not difficult to show that the extended modular group $\Gamma\left(Z_{c}\right)=\operatorname{Hom}\left(Z_{c}\right) / \operatorname{Hom}_{0}^{+}\left(Z_{c}\right)$ is the full group of holomorphic or antiholomorphic automorphisms of $T\left(Z_{c}\right)$ (cf. [6], 5.10, page 35). Especially the mapping $\sigma^{*}: T\left(Z_{c}\right) \rightarrow T\left(Z_{c}\right)$ is an antiholomorphic involution.

By Fricke's theorem, $\Gamma^{+}\left(Z_{c}\right)$ acts properly discontinuously on $T\left(Z_{c}\right)$. Hence $X\left(Z_{c}\right)=T\left(Z_{c}\right) / \Gamma^{+}\left(Z_{c}\right)$ is an irreducible normal complex space.

Since $\sigma^{*}$ is an antiholomorphic involution, its fixed-point set $T\left(Z_{c}\right)_{\sigma^{*}}$ is a real analytic submanifold of $T\left(Z_{c}\right)$. Hence we can endow $T(Z)$ with a real analytic structure requiring $\pi^{*}: T(Z) \rightarrow T\left(Z_{c}\right)$ to be real analytic. Then the modular group $\Gamma(Z)$ consists of real analytic automorphisms of $T(Z) . T(Z)$ with this structure is a $3 g$-3-dimensional real analytic manifold. Recall that by classical methods we can show that $T(Z)$ is simply connected ([6], page 29).

\section{The real analytic structure of $X(Z)$}

Unfortunately, the natural mapping $\pi^{*}: X(Z) \rightarrow X\left(Z_{c}\right), \pi^{\cdot}([X])=\left[X_{c}\right]$, is generally not one-to-one. Hence we cannot employ the above method to define a real analytic structure on $X(Z)$. Let us consider a covering space of $X\left(Z_{c}\right)$.

Define $\operatorname{Hom}^{+}\left(Z_{c}, \sigma\right)=\left\{h \in \operatorname{Hom}^{+}\left(Z_{c}\right) \mid h \circ \sigma=\sigma \circ h\right\}$, and let $\Gamma^{+}\left(Z_{c}, \sigma\right)=$ $\operatorname{Hom}^{+}\left(Z_{c}, \sigma\right) / \operatorname{Hom}_{0}^{+}\left(Z_{c}, \sigma\right) . \Gamma^{+}\left(Z_{c}, \sigma\right)$ is the relative modular group of $Z_{c}$, and $\mathrm{Hom}^{+}\left(Z_{c}, \sigma\right)$ is just the group of orientation preserving liftings of homeomorphic self-mappings of $Z$, i.e., the group of those mappings $h: Z_{c} \rightarrow Z_{c}$ for which there 
exists a mapping $Z \rightarrow Z$ such that the diagram

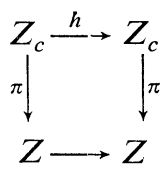

commutes.

The relative modular group $\Gamma^{+}\left(Z_{c}, \sigma\right)$ acts properly discontinuously on $T\left(Z_{c}\right)$, and the relative moduli space, $X\left(Z_{c}, \sigma\right)=T\left(Z_{c}\right) / \Gamma^{+}\left(Z_{c}, \sigma\right)$, is an irreducible normal complex space.

Consider now the mapping $\pi^{\prime}: X(Z) \rightarrow X\left(Z_{c}, \sigma\right), \pi^{\prime}([X])=\left[X_{c}\right]$. The diagram

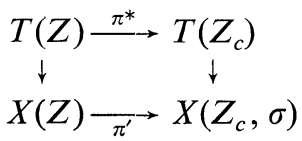

commutes, and $\pi^{\prime}$ defines a homeomorphism of $X(Z)$ onto its image in $X\left(Z_{c}, \sigma\right)$. Let us identify $X(Z)$ with $\pi^{\prime}(X(Z)) \subset X\left(Z_{c}, \sigma\right)$. The involution $\sigma: Z_{c} \rightarrow Z_{c}$ induces real structures $\sigma^{\prime}: X\left(Z_{c}, \sigma\right) \rightarrow X\left(Z_{c}, \sigma\right)$ and $\sigma^{\circ}: X\left(Z_{c}\right) \rightarrow X\left(Z_{c}\right)$. It is clear that $X(Z) \subset X\left(Z_{c}, \sigma\right)$ is the image of the real part $T\left(Z_{c}\right)_{\sigma^{*}}$ of $T\left(Z_{c}\right)$ under the projection $T\left(Z_{c}\right) \rightarrow X\left(Z_{c}, \sigma\right)$. Now we can apply Theorem 2, getting immediately:

Theorem 3. The moduli space $X(Z)$ is an irreducible real analytic space. It is contained in the quasiregular real part $X\left(Z_{c}, \sigma\right)_{\sigma^{*}}$ of the relative moduli space $X\left(Z_{c}, \sigma\right)$.

Theorem 4. The image of $X(Z)$ under the projection $X\left(Z_{c}, \sigma\right) \rightarrow X\left(Z_{c}\right)$ is an irreducible real analytic subspace of $X\left(Z_{c}\right)$. It is contained in the quasiregular real part, $X\left(Z_{c}\right)_{\sigma}$., of $X\left(Z_{c}\right)$.

Note that by Proposition $1 X(Z) \subset X\left(Z_{c}, \sigma\right)$ is locally irreducible.

The projection $X\left(Z_{c}, \sigma\right) \rightarrow X\left(Z_{c}\right)$ is not one-to-one, but if two different points $\left[X_{c}\right],\left[Y_{c}\right] \in X(Z) \subset X\left(Z_{c}, \sigma\right)$ are mapped onto one point in $X\left(Z_{c}\right)$, then - as one can easily check - the Riemann surfaces $X_{c}$ and $Y_{c}$ have non-trivial holomorphic self-mappings. Hence that one point lies, in general, in the singular part of $X\left(Z_{c}\right)$. Using Lemma 1 we can show that the image of $X(Z)$ in $X\left(Z_{c}\right)$ is the closure of its regular part. Hence, if we study the image of $X(Z)$ in $X\left(Z_{c}\right)$ instead of $X(Z)$ itself, we do not lose too many points. This motivates the considerations in the following section. 


\section{The moduli space of Klein surfaces of given genus}

Denote by INV $\left(Z_{c}\right)$ the set of orientation reversing involutions of $Z_{c}$. Each $\zeta \in \operatorname{INV}\left(Z_{c}\right)$ induces an antianalytic involution $\zeta^{*}$ of $T\left(Z_{c}\right)$. The fixed-point set of $\zeta^{*}, T\left(Z_{c}\right)_{\zeta^{*}}$, can be identified with the Teichmüller space $T\left(Z_{c} / \zeta\right)$.

Assume that $\zeta_{1}$ and $\zeta_{2}$ are homotopic involutions. Then

$$
\zeta_{1}^{*}=\left(\zeta_{1} \circ \zeta_{2}^{-1} \circ \zeta_{2}\right)^{*}=\left(\zeta_{1} \circ \zeta_{2}^{-1}\right)^{*} \circ \zeta_{2}^{*}=\zeta_{2}^{*},
$$

since $\zeta_{1} \circ \zeta_{2}^{-1} \in \mathrm{Hom}_{0}^{+}\left(Z_{c}\right)$. Hence homotopic involutions of $Z_{c}$ induce the same involution of the Teichmüller space $T\left(Z_{c}\right)$.

Let

$$
T(\boldsymbol{R}, g)=\left\{p \in T\left(Z_{c}\right) \mid \zeta^{*}(p)=p \text { for some } \zeta \in \operatorname{INV}\left(Z_{c}\right)\right\} .
$$

The set $T(\boldsymbol{R}, g)$ is the union of the Teichmüller spaces of all non-classical Klein surfaces of genus $g$ (=the genus of $Z_{c}$ ). If $T\left(Z_{c}\right)$ is interpreted as the Teichmüller space of complex algebraic curves, then $T(R, g)$ is the subspace of $T\left(Z_{c}\right)$ whose points can be defined by real polynomials. Note that $T(R, g)$ is a closed subset of $T\left(Z_{c}\right)$. This follows from the fact that the extended moduli group $\operatorname{Hom}\left(Z_{c}\right) /$ $\operatorname{Hom}_{0}^{+}\left(Z_{c}\right)$ acts properly discontinuously on $T\left(Z_{c}\right)$.

The image of $T(R, g)$ under the projection $T\left(Z_{c}\right) \rightarrow X\left(Z_{c}\right)$ is called the moduli space of non-classical Klein surfaces of genus $g$. We will denote it by $X(\boldsymbol{R}, g)$.

Let $\zeta_{1}$ and $\zeta_{2}$ be orientation reversing involutions of $Z_{c}$. They induce involutions $\zeta_{1}$ and $\zeta_{2}$ of $X\left(Z_{c}\right)$. Since $\zeta_{1} \circ \zeta_{2}^{-1} \in \mathrm{Hom}^{+}\left(Z_{c}\right)$, we have

$$
\zeta_{1}=\left(\zeta_{1} \circ \zeta_{2}^{-1} \circ \zeta_{2}\right)^{\cdot}=\left(\zeta_{1} \circ \zeta_{2}^{-1}\right)^{\cdot} \circ \zeta_{2}=\zeta_{2} \text {. }
$$

Hence all mappings of $\operatorname{INV}\left(Z_{c}\right)$ induce the same involution of the moduli space. Let $\sigma$ be the involution of $Z_{c}$ that was previously fixed in Section 4. Then we get, using Theorem 4 many times, the following result:

Lemma 2. The moduli space $X(\boldsymbol{R}, g)$ is contained in the quasiregular real part $X\left(Z_{c}\right)_{\sigma}$. of the moduli space $X\left(Z_{c}\right)$.

In the case $g>2$ we can find out explicitly what the irreducible components of $X(\boldsymbol{R}, g)$ are. To that end we need the following simple result.

Lemma 3. Assume that $g>2$ and that the point $[X] \in T\left(Z_{c}\right)$ is not fixed by any non-identity element of the moduli group $\Gamma^{+}\left(Z_{c}\right)$. Then the Riemann surface $X$ does not have any other holomorphic self-mappings than the identity mapping.

Proof. Let $[X] \in T\left(Z_{c}\right)$ be a point which is not fixed by any non-identity mapping of the modular group. Assume that there exists a $g \in \mathrm{Hom}^{+}\left(Z_{c}\right)$ such that $g: X \rightarrow X$ is holomorphic. If $g$ is not the identity mapping, it is not even homotopic to the identity mapping. Let us assume that such is the case, and let us show that this is not possible. 
Since $g: X \rightarrow X$ is holomorphic, the mapping $g^{*}: T\left(Z_{c}\right) \rightarrow T\left(Z_{c}\right)$ keeps the point $[X]$ fixed. Since $[X]$ is not fixed by any non-identity mapping of the modular group, $g^{*}$ must be the identity mapping of $T\left(Z_{c}\right)$.

Let $[Y] \in T\left(Z_{c}\right)$ be an arbitrary point. Since $g^{*}$ is the identity mapping of $T\left(Z_{c}\right),[Y]=\left[g^{*}(Y)\right]$, and we can find a holomorphic mapping $h: g^{*}(Y) \rightarrow Y$ which belongs to the group $\operatorname{Hom}_{0}^{+}\left(Z_{c}\right)$. Then the mapping $h \circ g$ is a holomorphic self-mapping of the Riemann surface $Y$. Since $g \notin \operatorname{Hom}_{0}^{+}\left(Z_{c}\right)$ and $h \in \operatorname{Hom}_{0}^{+}\left(Z_{c}\right)$, the composed mapping $h \circ g$ is not homotopic to the identity mapping. Hence the Riemann surface $Y$ has non-trivial holomorphic self-mappings. But a generic compact Riemann surface of genus $>2$ does not have any non-trivial holomorphic self-mappings. Hence we have reached a contradiction, since $Y \in K^{+}\left(Z_{c}\right)$ was arbitrary. This proves the lemma.

Let us now consider irreducible components of $X(\boldsymbol{R}, g)$. Assume $\zeta_{1}$ and $\zeta_{2}$ are such involutions of $Z_{c}$ that the intersection of the images of $T\left(Z_{c}\right) \xi_{1}^{*}$ and $T\left(Z_{c}\right)_{\xi^{*}}$ in $X\left(Z_{c}\right)$ contains a non-empty open subset of $X(\boldsymbol{R}, g)$. Then, by Lemma 1, we can find points $[X] \in T\left(Z_{c}\right)_{\xi_{1}^{*}}$ and $[Y] \in T\left(Z_{c}\right)_{\xi_{2}^{*}}$ which are mapped onto the same point in $X\left(Z_{c}\right)$ and which are not fixed by any non-identity element of the moduli group $\Gamma^{+}\left(Z_{c}\right)$. We may assume that $\zeta_{1}: X \rightarrow X$ and $\zeta_{2}: Y \rightarrow Y$ are antiholomorphic.

Since the Riemann surfaces $X$ and $Y$ correspond to the same point in the modular space $X\left(Z_{c}\right)$, we can find a holomorphic mapping $g: X \rightarrow Y$. The mapping $\zeta_{2}^{-1} \circ g^{-1} \circ \zeta_{2} \circ g$ is then a holomorphic self-mapping of the Riemann surface $X$. Since $[X] \in T\left(Z_{c}\right)$ is not fixed by any non-identity element of the modular group, $X$ does not have non-trivial holomorphic self-mappings. Hence $\zeta_{1}^{-1} \circ g^{-1} \circ \zeta_{2} \circ g$ is the identity mapping, i.e.,

$$
g \circ \zeta_{1}=\zeta_{2} \circ g .
$$

It follows that the mapping $g: X \rightarrow Y$ induces a dianalytic homeomorphism between the Klein surfaces $X / \zeta_{1}$ and $Y / \zeta_{2}$. Consequently, $X / \zeta_{1}$ and $Y / \zeta_{2}$ are of the same topological type.

On the other hand, it is easy to see that if two involutions $\zeta_{1}$ and $\zeta_{2}$ are such that the corresponding Klein surfaces are of the same topological type, the images in $X\left(Z_{c}\right)$ of the fixed-point sets $T\left(Z_{c}\right)_{\xi_{1}^{* 1}}$ and $T\left(Z_{c}\right)_{\xi_{2}^{*}}$ coincide.

These considerations in conjunction with Theorem 4 yield the following result:

Theorem 5. The moduli space $X(\boldsymbol{R}, g)$ of non-classical Klein surfaces of genus $g, g>1$, is a real analytic subset of $X\left(Z_{c}\right)$, and is contained in the quasiregular real part $X\left(Z_{c}\right)_{\sigma}$. of $X\left(Z_{c}\right)$. If $g>2$, the irreducible components of $X(\boldsymbol{R}, g)$ correspond to Klein surfaces of a given topological type, and $X(\boldsymbol{R}, g)$ has exactly

$$
2[g / 2]+[(g+1) / 2]+2
$$

irreducible components. 
Proof. The only point that we have not proved yet is the formula for the number of irreducible components of $X(\boldsymbol{R}, g)$. But that is the same as the number of different topological types of non-classical Klein surfaces of genus $g$. Hence a computation that R. Zarrow has done ([7], Lemma in Chapter 2) gives the above result.

In the case $g>3$ it turns out that $X(\boldsymbol{R}, g)$ coincides with the quasiregular real part of $X\left(Z_{c}\right)$. In order to prove that let us first show that $X(\boldsymbol{R}, g)$ is a closed subset of $X\left(Z_{c}\right)$.

To that end assume that $p_{1}, p_{2}, \ldots$ is a sequence in $X(\boldsymbol{R}, g)$ which converges to a point $p_{0} \in X\left(Z_{c}\right)$. It suffices to show that $p_{0} \in X(\boldsymbol{R}, g)$.

We can choose a point $\tilde{p}_{0} \in T\left(Z_{c}\right)$ lying over $p_{0} \in X\left(Z_{c}\right)$ and a sequence $\tilde{p}_{1}, \tilde{p}_{2}, \ldots \in T\left(Z_{c}\right)$ converging to $\tilde{p}_{0}$ such that each $\tilde{p}_{i}$ belongs to $T(\boldsymbol{R}, g)$ and lies over the point $p_{i}$ in $X(\boldsymbol{R}, g)$. Hence every point $\tilde{p}_{i}$ is fixed by some involution $\zeta_{i}^{*}$ of $T\left(Z_{c}\right)$. Since the extended modular group Hom $\left(Z_{c}\right) / \operatorname{Hom}_{0}^{+}\left(Z_{c}\right)$ acts properly discontinuously on $T\left(Z_{c}\right)$, it follows that also $\tilde{p}_{0}$ must be fixed by some involution $\zeta^{*}$. Hence $p_{0} \in X(\boldsymbol{R}, g)$, and $X(\boldsymbol{R}, g)$ is closed.

Let $S$ denote the singular set of the complex space $X\left(Z_{c}\right)$. As we have previously noted, the quasiregular real part $X\left(Z_{c}\right)_{\sigma}$. is the closure of $X\left(Z_{c}\right)_{\sigma} \backslash \backslash S$. Hence if we can show that $X\left(Z_{c}\right)_{\sigma} \backslash \backslash S \subset X(\boldsymbol{R}, g)$, we have in fact proved that $X\left(Z_{c}\right)_{\sigma}=X(\boldsymbol{R}, g)$. To prove the above inclusion choose a point $[Y] \in X\left(Z_{c}\right)_{\sigma} . \backslash S$. Since $[Y] \notin S$, and since the genus of $Y$ is $>3$, the Riemann surface $Y$ does not have non-trivial holomorphic self-mappings. This follows from Theorem 1 in [5].

On the other hand, since $[Y]$ is a fixed point of the involution $\sigma^{\circ}$ of $X\left(Z_{c}\right)$, we can find a holomorphic mapping $g: \sigma^{*}(Y) \rightarrow Y$. Recall that by definition $\sigma^{*}([Y])=$ $\left[\sigma^{*}(Y)\right]$, where $\sigma^{*}(Y)$ is the analytic structure of $Z_{c}$ for which the mapping $\sigma: Y \rightarrow \sigma^{*}(Y)$ is antiholomorphic. Then $g \circ \sigma$ is an antiholomorphic self-mapping of the Riemann surface $Y$. Since $Y$ does not have holomorphic self-mappings other than the identity mapping, $g \circ \sigma$ is an involution. It follows that $[Y] \in X(\boldsymbol{R}, g)$. This proves the inclusion $X\left(Z_{c}\right)_{\sigma} . \backslash S \subset X(\boldsymbol{R}, g)$. On the grounds of the previous remark, we have now proved the following theorem:

The orem 6. The moduli space of non-classical Klein surfaces of genus $g, g>3$ is the quasiregular real part of the moduli space of Riemann surfaces of genus $g$.

Remark 1. An example of Earle ([4], Theorem 2, p. 125) shows that the involution $\sigma^{*}: X\left(Z_{c}\right) \rightarrow X\left(Z_{c}\right)$ actually has fixed points that do not belong to $X(R, g)$, but Theorem 6 shows that the dimension of the set of such points is, in general, lower than the dimension of $X(\boldsymbol{R}, g)$.

Remark 2. It is not clear whether the statement of Theorem 6 holds in the case $g=2$ or $g=3$, because for those genera there exist hyperelliptic Riemann surfaces that have non-trivial holomorphic automorphisms but do not sit in the singular part of the moduli space. 
Question. Is $\sigma^{*}: X\left(Z_{c}\right) \rightarrow X\left(Z_{c}\right)$ the only antiholomorphic automorphism of the moduli space $X\left(Z_{c}\right)$ ?

Another question. Is the moduli space $X(\boldsymbol{R}, g)$ connected?

\section{References}

[1] Alling, N. L., and N. Greenleaf: Klein surfaces and real algebraic function fields. - Bull. Amer. Math. Soc. 75, 1969, 869-872.

[2] Alling, N. L., and N. Greenleaf: Foundations of the theory of Klein surfaces. - Lecture Notes in Mathematics 219, Springer-Verlag, Berlin-Heidelberg-New York, 1971.

[3] Andreotti, A., and P. Holm: Quasianalytic and parametric spaces. - Real and complex singularities, Oslo 1976, Proceedings of the Nordic Summer School/NAVF, Symposium in Mathematics, Oslo, August 5-25, 1976, edited by P. Holm, Sijthoff \& Noordhoff International Publishers, Alphen aan den Rijn, The Netherlands, 1977.

[4] EARLe, C. J.: On the moduli of closed Riemann surfaces with symmetries. - Advances in the theory of Riemann surfaces, Annals of Mathematics Studies 66, Princeton University Press and University of Tokyo Press, Princeton, New Jersey, 1971, 119-130.

[5] RaUch, H. E.: The singularities of the modulus space. - Bull. Amer. Math. Soc. 68, 1962, 390394.

[6] SEPpäLÄ, M.: Teichmüller spaces of Klein surfaces. - Ann. Acad. Sci. Fenn. Ser. A I Math. Dissertationes 15, 1978, 1-37.

[7] ZARrow, R.: On Earle's mod $n$ relative Teichmüller spaces. - Canad. Math. Bull. 21, 1978, $355-360$.

Helsinki School of Economics

Department of Methodological Sciences

SF-00100 Helsinki 10

Finland

Received 22 October 1979 